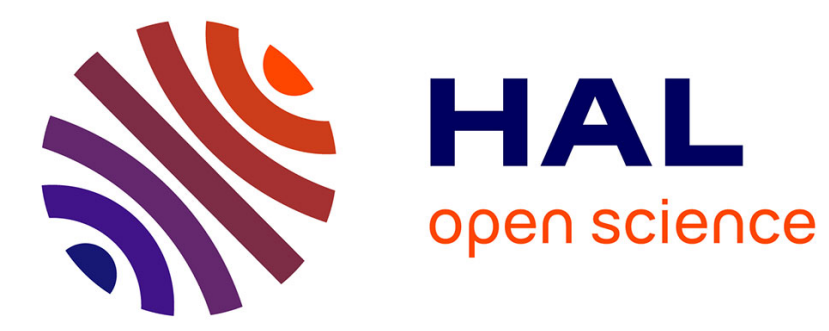

\title{
Stable and Optimal Interface Treatment for Partitioned Conjugate Heat Transfer Problems
}

\author{
Marc-Paul Errera, Rocco Moretti, Yohann Bachelier, Tristan Soubrié
}

\section{To cite this version:}

Marc-Paul Errera, Rocco Moretti, Yohann Bachelier, Tristan Soubrié. Stable and Optimal Interface Treatment for Partitioned Conjugate Heat Transfer Problems. AIAA Scitech 2019 Forum, Jan 2019, SAN DIEGO, United States. 10.2514/6.2019-1561 . hal-02908793

\section{HAL Id: hal-02908793 https://hal.science/hal-02908793}

Submitted on 29 Jul 2020

HAL is a multi-disciplinary open access archive for the deposit and dissemination of scientific research documents, whether they are published or not. The documents may come from teaching and research institutions in France or abroad, or from public or private research centers.
L'archive ouverte pluridisciplinaire HAL, est destinée au dépôt et à la diffusion de documents scientifiques de niveau recherche, publiés ou non, émanant des établissements d'enseignement et de recherche français ou étrangers, des laboratoires publics ou privés. 


\title{
Stable and Optimal Interface Treatment for Partitioned Conjugate Heat Transfer Problems
}

\author{
Marc-Paul Errera, ${ }^{1}$ Rocco Moretti ${ }^{2}$, Yohann Bachelier ${ }^{3}$ \\ DAAA-NFLU, ONERA, Université Paris Saclay, Châtillon, France 99232 \\ and \\ Tristan Soubrié ${ }^{4}$ \\ Andheo 29 Av. Division Leclerc, 99232 Châtillon, France
}

\begin{abstract}
In conjugate heat transfer (CHT) analysis, the fluid-solid heat transfer calculation is realized by expanding the fluid capability to include heat conduction in solid regions neighboring the fluid. Stability conditions of CHT analysis can be provided by a simplified model problem and from this model, two fundamental parameters are introduced in the coupled approach : a "numerical" Biot number, and an optimal coefficient. The first parameter defines the nature of the fluid-solid interaction. The optimal coefficient ensures unconditional stability. Results presented in this paper consider a wide range of steady thermal phenomena from weak to very strong fluid-solid interaction (insulating materials). At the end of the paper, an example of transient CHT is also shown. The methods proposed in this paper illustrate that a model problem can provide effective and practical solutions directly applicable to complex conjugate heat transfer problems.
\end{abstract}

\section{Nomenclature}

$\begin{array}{ll}a & =\text { thermal diffusivity, } \mathrm{m}^{2} / \mathrm{s} \\ B i^{(\Delta)} & =\text { mesh Biot number } \\ B i_{v} & =\text { numerical Biot number } \\ D & =\text { Fourier number } \\ \bar{D} & =\text { normalized Fourier number } \\ h & =\text { coupling coefficient, } \mathrm{Wm}^{-2} \mathrm{~K}^{-1} \\ K & =\text { thermal conductance, } \mathrm{Wm}^{-2} \mathrm{~K}^{-1} \\ q & =\text { heat flux, } \mathrm{Wm}^{-2} \\ T & =\text { temperature, } \mathrm{K} \\ \alpha & =\text { coupling coefficient, } \mathrm{Wm}^{-2} \mathrm{~K}^{-1} \\ \lambda & =\text { thermal conductivity, } \mathrm{Wm}^{-1} \mathrm{~K}^{-1} \\ \Delta y & =\text { size } 1^{\text {st }} \text { cell, } \mathrm{m}\end{array}$

\footnotetext{
Engineer, Ph.D., ONERA-DAAA/NFLU. Member AIAA.

Ph.D. Student, ONERA-DAAA/NFLU.

Ph.D. Student.

Head Manager, Andheo, Ph.D.
} 


\section{Introduction}

C Conjugate heat transfer (CHT) is employed to analyze thermal interaction processes at a fluid-solid interface. Heat transfer is of fundamental importance in many fluid dynamics applications [1][2] since flows are often confined within some material. Moreover, heat is in general transferred and change the flow properties in a non-trivial way. As a result, a coupled approach is the most accurate way to implement when all boundary conditions and thermal state of a device are interactively evaluated. It is the reason why, CHT, also called thermal fluid-solid interaction (FSI) has become an important subfield of numerical simulation. In recent years, many papers were published concerning the numerical modeling of CHT problems and many interesting stability studies are available [3][4][5][6][7][8][9][10].

Recently, we have shown using a 1D thermal model problem that in a coupled system, a numerical transition can be identified [11][12]. This fundamental result has been derived from a normal mode stability analysis based on the theory of Godunov-Ryabenkii [13] applied to a model problem. This transition can be regarded as an optimal choice in terms of stability and convergence. The performance of this method was tested in an industrial furnace and the relevance of this model was fully confirmed [14]. On this mathematical basis, the influence of the main numerical and physical parameters can be understood and evaluated.

This paper presents the main results of this model problem with a Dirichlet-Robin interfacial procedure. The model higlights two fundamental parameters, a "numerical" Biot number and an optimal coupling coefficient. The first parameter determines the nature (weak, moderate, strong) of the fluid-solid interaction The optimal coefficient ensures, at least theoretically, unconditional stability. However, in the case of strong FSI, numerical stability issues may arise. The final part of the paper will focus on unsteady CHT and it will be shown that the model problem can also provide efficient solutions for obtaining stable coupled computations.

\section{Dirichlet-Robin Interface treatment}

\section{A. Partitioned approach}

The simulation of multiphysics problems, i.e. fluid-solid interaction, is generally accomplished by partitioned staggered schemes [15][16][17]. Each system is treated by discretization techniques with algorithms that are known to perform well individually. As a result, a stable fluid-solid solution will be sought for steady CHT by coupling a transient fluid solution with a steady solid state.

\section{B. Model problem}

The nature of instabilities derived from a 1D model problem can give insight into the potential instabilities in 2D/3D computations. Thus, the behavior of interface conditions in CHT is often studied using a normal mode analysis applied to a $1 \mathrm{D}$ model. This model is composed of two partitions with a shared interface.

Interface conditions are needed on either side of the shared interface, where coupling conditions are applied. Our goal is to ensure a stable CHT process and to avoid destabilizing effects. It is well known that Robin conditions have many attractive features and thus a Robin condition is applied to the solid side

$$
\hat{q}_{s}+\alpha_{f} \hat{T}_{s}=q_{f}+\alpha_{f} T_{f}
$$

The subscripts $f$ and $s$ denote the fluid and solid domain respectively and the $\left({ }^{\wedge}\right)$ notation indicates the sought values. $q$ is the interface heat flux and $T$ is the interface temperature. The general Robin condition (1) introduces the numerical coupling parameter $\alpha_{f}$ the choice of which directly influences the stability of the coupling process. On the fluid side, a Dirichlet condition is imposed:

$$
\hat{T}_{f}=T_{s}
$$

\section{Amplification factor and mesh Biot number}

The Godunov-Ryabenkii (G-R) stability analysis is very similar to the standard Fourier stability method except that the Fourier analysis ignores boundary conditions. A normal mode solution is applied to the case defined by the equations in the discrete model problem, and, after elementary transformations, we obtain (see [11][12] for more details) the following temporal amplification factor 


$$
g\left(z, \alpha_{f}\right)=\frac{1}{K_{s}+\alpha_{f}}\left[\kappa_{f}\left(\alpha_{f}, D\right)+\alpha_{f}-K_{f}\right]
$$

Where $a_{f}$ is the thermal diffusivity and $\kappa_{f}$ is the "spatial" amplification factor that depends on $\alpha_{f}$ and on the Fourier number $D$, expressed by

$$
D=a_{f} \Delta t / \Delta x_{f}^{2}
$$

$K_{f}$ and $K_{s}$ are the fluid and solid conductances respectively. The ratio of these two conductances can thus be defined :

$$
B i^{(\Delta)}=\frac{K_{f}}{K_{s}}
$$

and this dimensionless number can be regarded as a mesh Biot number.

\section{Numerical Biot number : Nature of the thermal Fluid-Structure Interaction}

The CHT model is stable in the sense of G-R if $\left|g\left(z, \alpha_{f}\right)\right|<1$. This condition applied to (3) leads, after some basic calculus manipulations and after introducing a normalized Fourier number $\bar{D}_{f}$, to two zones :

- Weak interaction : $B i_{v}=B i^{(\Delta)}\left(1-\bar{D}_{f}\right) \leq 1$. The coupling process is stable $\forall \alpha_{f} \geq 0$. If this condition holds, the "transient" thermal resistance of the fluid domain at the shared interface is greater than the resistance offered by the whole solid domain. A Dirichlet condition on the fluid side is therefore appropriate.

- Moderate/Strong interaction : $B i_{v}=B i^{(\Delta)}\left(1-\bar{D}_{f}\right) \geq 1$. The coupling procedure exhibits a lower stability bound $\alpha_{f}^{\min }$ and this implies either that the solid thermal gradients are not negligible or that the thermal fluid conductance is larger than that of the solid.

This demonstrates how stability depends mainly on the ratio of thermal resistances, but also on the dynamics of the transient fluid system. The higher the local Biot number, the more difficult it will be to stabilize the coupling.

\section{E. Optimal coefficient}

It is noteworthy that the modulus of the amplification factor does not have a monotonic variation (in terms of $\alpha_{f}$ ) but goes through an absolute minimum, denoted $\alpha_{f}^{\text {opt }}$. In other words, the existence of a transition value for $\alpha_{f}$ can be identified. At this transition value, the shape of the curve of the amplification factor switches and turns back as can be seen in Figure 1.

This transition occurs at a unique and remarkable value $\alpha_{f}^{o p t}$ whose exact expression is given by

$$
\alpha_{f}^{o p t}=\frac{K_{f}}{2}\left(1-\bar{D}_{f}\right)
$$

When the optimal value defined by (6) is employed, we obtain the best-case scenario with no additional computational effort. The point $\alpha_{f}^{o p t}$ is the intersection of two opposite zones. The left half-line $\left(\alpha_{f}<\alpha_{f}^{o p t}\right)$ is a fast process prone to instability. The right half-line $\left(\alpha_{f}>\alpha_{f}^{o p t}\right)$ is a low but always stable process. The intersection $\alpha_{f}^{\text {opt }}$ is a perfect equilibrium between both. The role of $\alpha_{f}^{\text {opt }}$ in controlling and guiding the behavior of the CHT process is fundamental. Figure 1 illustrates three types of interaction. The amplification factor is represented $v s$ the coupling coefficient. The left curve can be totally inside the stability zone (weak interaction), partially outside (moderate interaction) or mainly outside (strong interaction). 


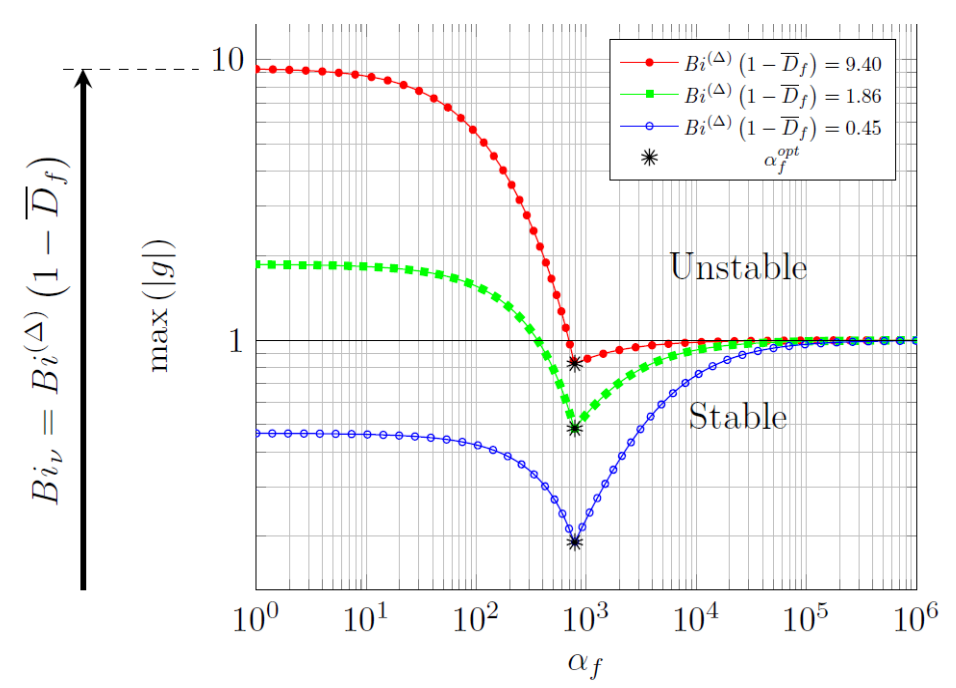

Fig. 1 Temporal amplification factor of Dirichlet-Robin condition for three numerical Biot numbers Blue curve : weak FSI. Green curve : moderate FSI. Red curve : strong FSI.

\section{Steady CHT computations in an academic test case}

\section{A. Numerical tools}

In the following, computing results are presented to illustrate the importance of the interface treatment in CHT problems. The numerical procedure is applied to the problem of convective heat transfer over, and conduction heat transfer within, a flat plate (see [18] for details). The fluid code, referred to as the elsA software package, is the ONERA multi-purpose tool for applied aerodynamics and multi-physics, which capitalizes on the innovative results of CFD research [19][20][21]. The solid software package, called Zset, is a comprehensive suite of integrated analysis programs for general purpose structural analysis [22]. The exchange of data between the two aforementioned solvers is carried out through the CWIPI library [23]. This library takes into account the grids, as well as the processes in which the data are located.

\section{B. Residuals and fluctuations}

Convergence, expressed by the decay of residuals, is fundamental but it can mask the fluctuations that inevitably occur during the coupling. Therefore, we have decided to look precisely at the amplitude of these fluctuations which are expressed by a Reynolds-averaged procedure.

\section{Weak interaction}

Weak interaction is considered here by implementing a solid conductivity such as to obtain a numerical Biot number $B i_{v}=0.5$, i.e. lower than unity. The convergence history for four values of the coupling coefficient, $\alpha_{f}$, is shown in Figure 2 by means of the interface temperature residual plotted as a function of the coupling iteration. In weak FSI, there are no significant differences for low values of the coupling coefficient $\alpha_{f}$ and it is seen that the trends marked by the two lines $\alpha_{f}=0$ and $\alpha_{f}=\alpha_{f}^{o p t}$ are roughly the same. For high values of the coupling coefficient, convergence is a little slower, but at a tolerable level. 


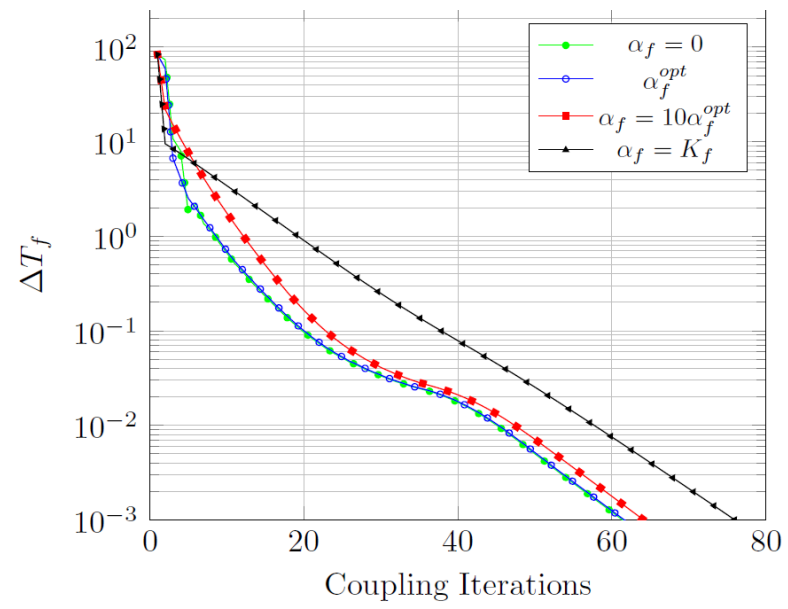

Fig. 2 Convergence history for weak thermal FSI and for 4 coupling coefficients.

Figure 3 shows simultaneously residuals and fluctuations $v s$ the coupling coefficient, for a weak FSI $\left(B i_{v}=1.07\right)$.
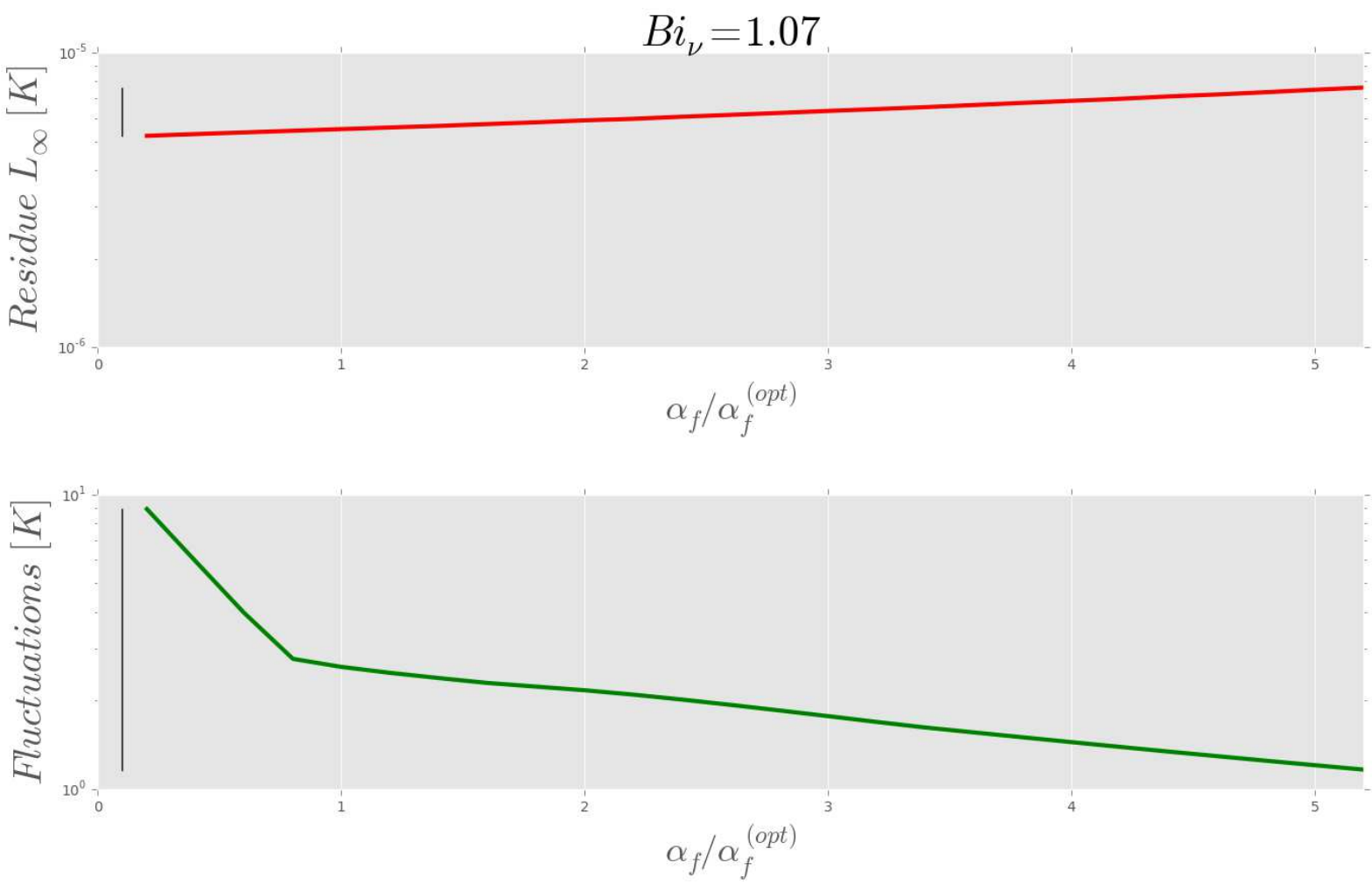

Fig. 3 Residuals and fluctuations for weak thermal FSI.

In this case the CHT process is stable for any coupling coefficient. The resisuals also show that the fluctuations intensity is a decreasing function of the coupling coefficient. The slope of this process is very slow (from $4.10^{-6}$ for $\alpha_{f} \approx 0$ to $6.10^{-6}$ for $\alpha_{f}=5 \alpha_{f}^{o p t}$ ). 


\section{Moderate interaction}

Moderate interaction has been considered by implementing a solid conductivity such as to obtain a numerical Biot number $B i_{v}=2.67$. The results are roughly the same as previously, however the main difference is that the CHT process is unstable for a low coupling coefficient. The fluctuations are always decreasing but they become acceptable, only around the value of the optimal coefficient. Greater values of the coupling coefficient slow down the CHT process.
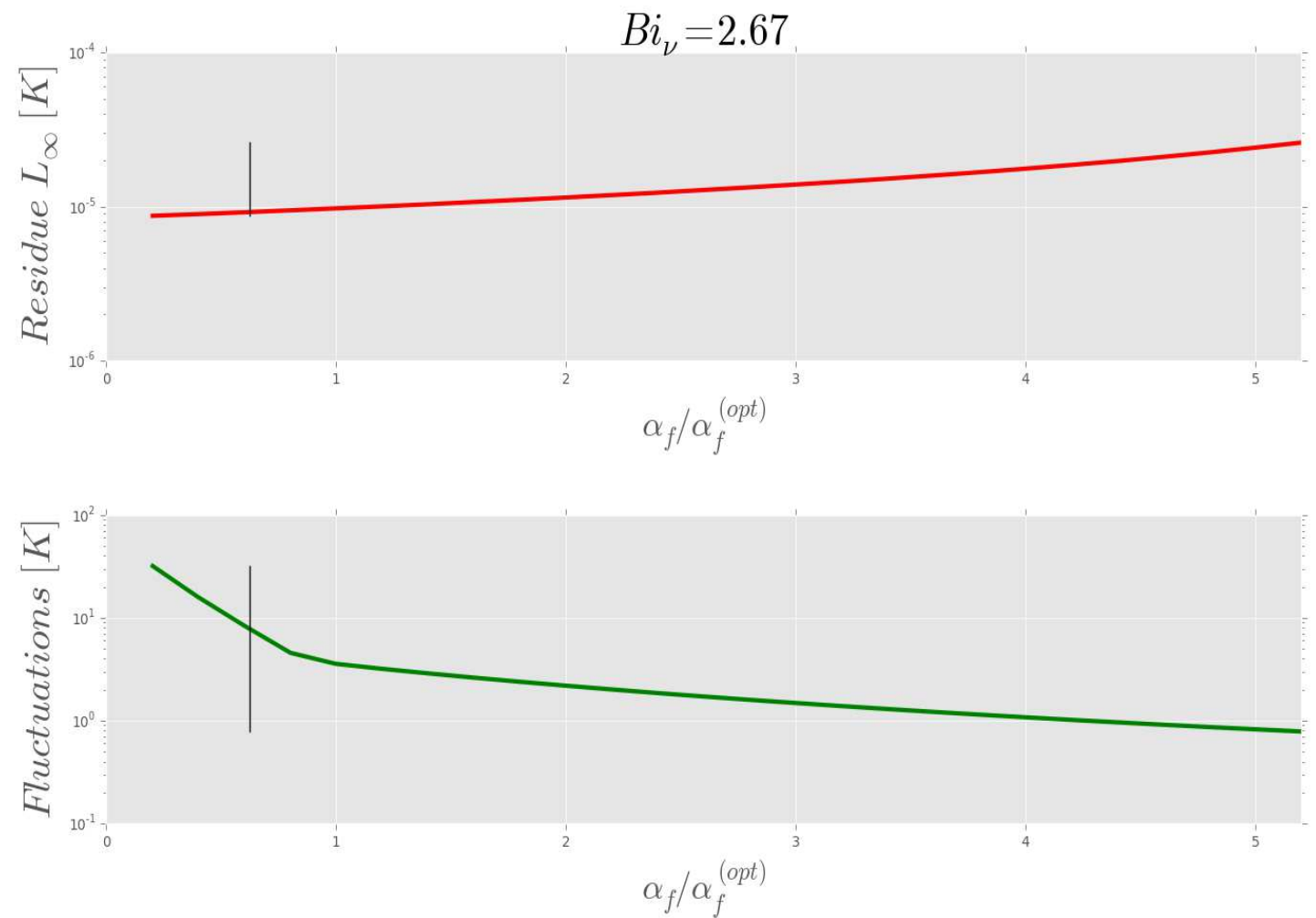

Fig. 4 Residuals and fluctuations for weak/moderate thermal FSI.

\section{E. Strong and stable interaction}

Unsurprisingly, at strong thermal FSI, the residuals are very large and the coupled simulation, as shown in Figure 5, is unstable when the coupling coefficient is small. The residuals decrease drastically and are ideal about the optimal coefficient. The CHT process becomes stable and the fluctuations are reasonable. This highlights the importance of this remarkable coefficient. When the coupling coefficient is large, the residuals decrease slowly, in a stable or overstable process, that is to say accompanied by small fluctuations. This demonstrates that a relevant CHT methodology is a trade-off between a fast but potentially unstable computation (low coefficient) and a stable process which can be quite slow. 

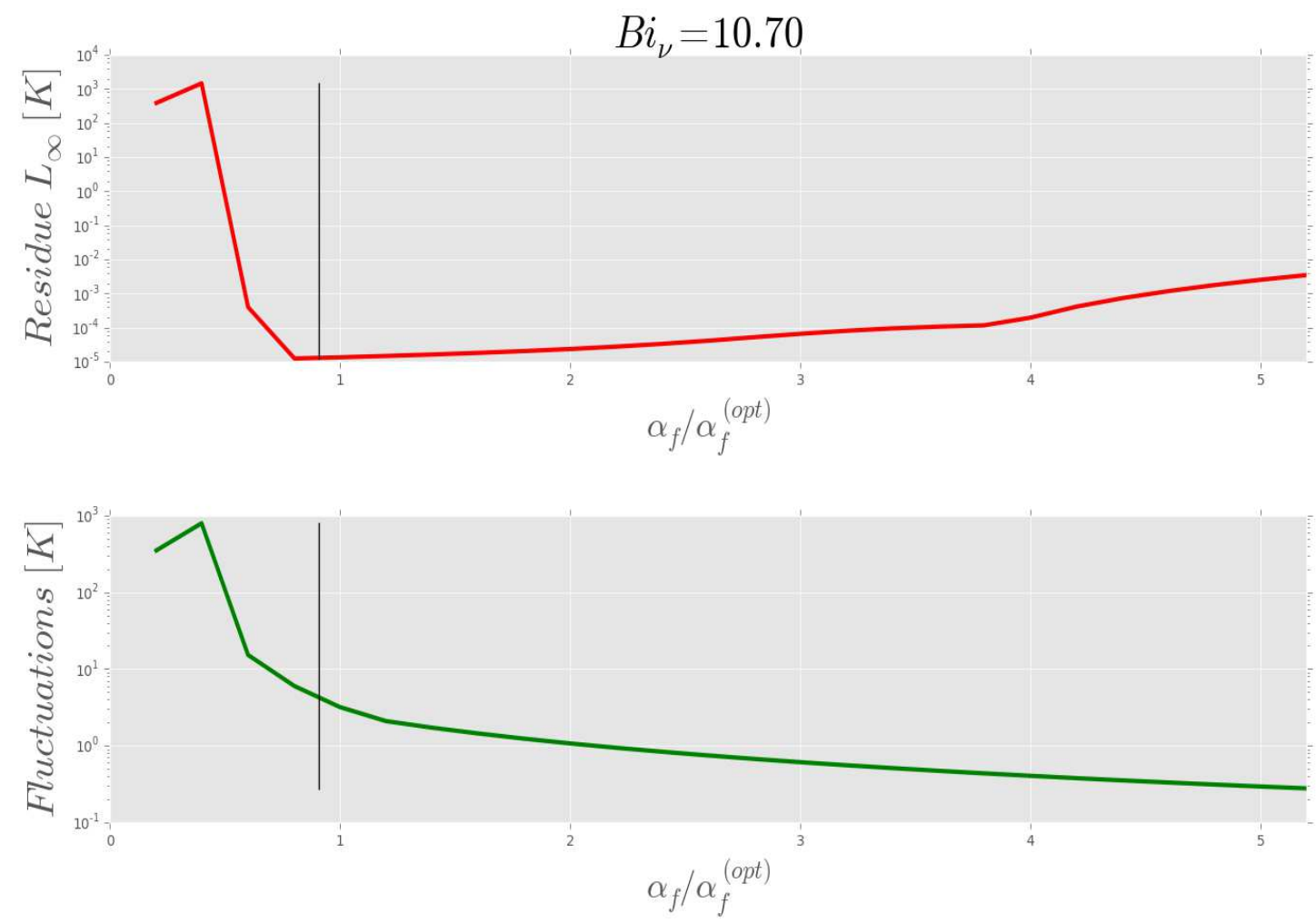

Fig. 5 Residuals and fluctuations for strong thermal FSI.

\section{F. Very strong interaction}

As the numerical Biot number increases further, the optimal Dirichlet-Robin does not achieve convergence (the temperature oscillates with a very large amplitude). As a result, a Neumann-Dirichlet interface condition has been considered here (heat flux imposed on the fluid side, temperature prescribed on the solid side). This is clearly the most physics-based procedure for a large Biot number, in other words when thermal gradients are significant in the solid material (strong thermal interaction problems). Figure 6 shows that convergence is obtained after 104 iterations. This is a satisfactory result, just slightly less than the one obtained with an optimal approach at weak or moderate FSI.

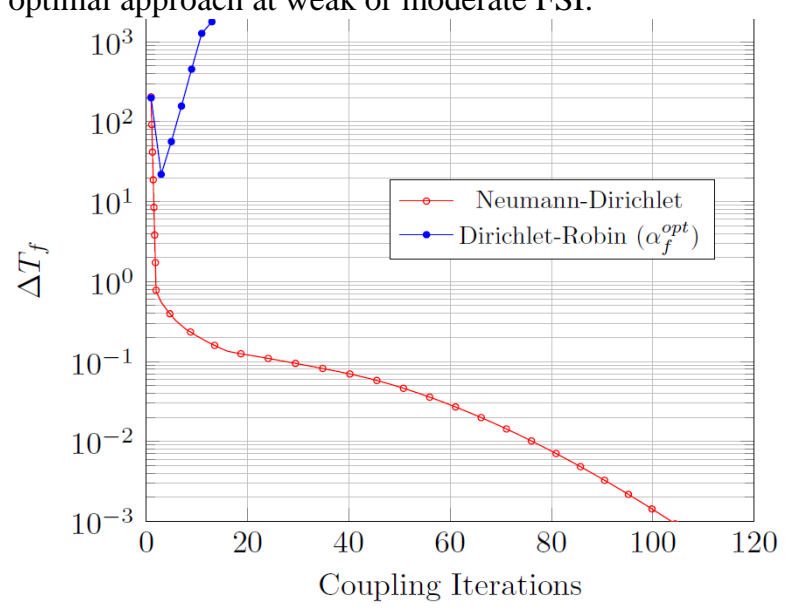

Fig. 6 Convergence history for very strong thermal FSI - The optimal coefficient of Dirichlet-Robin procedure and the Neumann-Robin procedure have been considered. 
As mentioned above, the Neumann-Dirichlet condition needs a number of iterations around $25 \%$ higher than a Dirichlet-Robin condition (104 iterations required to converge instead of 83 iterations) and with a smaller sensibility, the gap between the two coupling computations grows. However, it is important to stress that contrary to the cases using a Dirichlet-Robin condition, all the computations based on a Neumann condition are not prone to instabilities either when the solid conductivity or the coupling time step are getting smaller.

\section{Unsteady CHT computations: cooling of a turbine disk}

\section{A. Quasi-steady assumption}

The optimal schemes just presented in the case of a steady CHT can likewise be applied to transient coupling. Transient coupling is beginning to be employed in turbomachinery applications to account for the time-dependent thermal response of structures to ambient conditions. This approach is expected to grow rapidly since the transient heat load could lead to substantial gains in engine performance and component reliability. In such a context, the aim of unsteady CHT is to define relevant interfacial conditions of a twoway coupling procedure for practical CHT applications during a full transient flight cycle. This cycle is characterised by a long period of time, that is, the entire duration of a flight. This is needed since high requirements about the life-span of the engine elements are needed, in particular heat load characteristics during all the stages of a flight.

A computationally cheap procedure is to account for the time scale disparities between the fluid and solid domains and thus consider the flow solution as a sequence of steady states. The resulting "quasisteady assumption" is valid since the fluid and the solid operate on different time constants. This means that the influence of unsteadiness in the fluid domain is negligible and thus the flow solution is considered as a sequence of steady states. On the contrary, the solid is allowed to evolve over time.

\section{B. Relevant interface conditions}

The key point remains the choice of relevant interface conditions since they have a direct impact on numerical properties of the coupling methodology. However, the numerical expressions presented are devoted to steady CHT solutions only, and it is not possible to employ them directly. Another stability analysis adapted to transient problems is necessary. In effect, steady and unsteady CHT have very little in common. This analysis was carried out in another work [24] and only the expression of the optimal coefficient, in Dirichlet-Robin procedure, is provided here :

$$
\alpha_{f}^{o p t}=\frac{2 h}{1-\bar{D}_{s}}
$$

where $h$ is the heat transfer coefficient and $\bar{D}_{s}$ is the normalized Fourier number as expressed in the first part of this paper. However, note that this dimensionless number is, this time, defined in the transient solid domain.

It should be emphasized that the "steady strategy" in which only a coupled steady state is sought (first part of the current paper) is considerably different from that in the transient numerical approach constructed from the "quasi-steady-assumption". For instance, the Dirichlet-Robin procedure proposed for the first time in [11] based on the "optimal coefficient" (see Eq. 6) is radically different in several ways with the Dirichlet procedure where the influence of unsteadiness in the fluid domain is negligible (see Eq. 7). In the latter case, it can be seen that the optimal coefficient corresponds to the quotient between $2 h$ and a solid transient effect (term $\left.\left(1-\bar{D}_{s}\right)\right)$.

\section{An example}

Figure 7 depicts the temperature contours of a fluid-solid coupled system represented by an HP turbine disk and a cooling circuit on the left. This configuration has been used to account for the time-dependent thermal response of the disk structure over a long period of time (several hours). A two-way coupling of a dynamic thermal modeling in the solid and a sequence of fluid steady states has been considered. The most important point in this transient calculation is the choice of relevant interface conditions. It has been shown that when a Dirichlet-Robin procedure is employed, the coupling coefficient defined by (7) provides the best-performing method. 


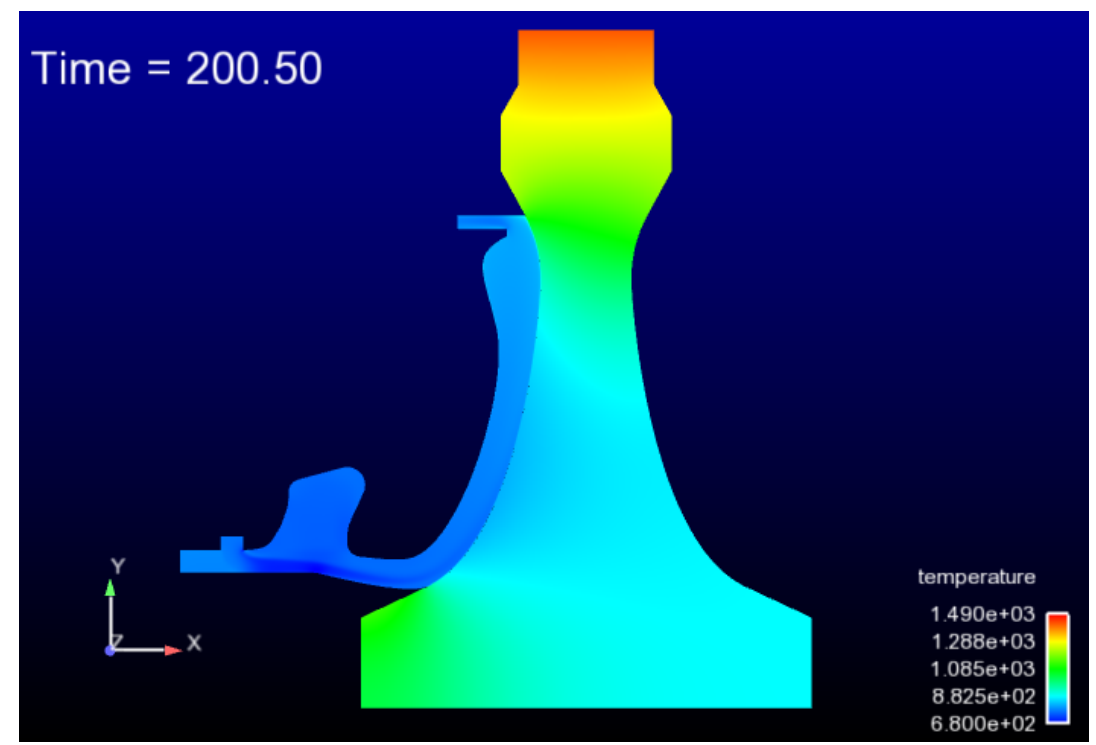

Fig. 7 Temperature contours in an HP turbine disk \& cooling circuit.

\section{Conclusion}

The CHT computations highlight the importance of a relevant transmission procedure taking into account the heterogeneous nature of complex systems. It has been shown that a so-called numerical Biot number represents a criterion to determine locally the nature of the thermal fluid-structure interaction. This number plays a key role in the stability process. From there, it is rather easy to analyze the effect and the influence of any parameter involved in CHT problems.

The tests in steady FSI have illustrated that a Dirichlet-Robin condition can be used ideally for low or moderate fluid-solid interaction since the optimal coefficient can turn the coupling process into an unconditionally stable method providing solutions quickly and effectively. However, for strong or very strong fluid-structure interaction, in other words when thermal gradients within the solid become important a Neumann condition imposed on the fluid side provides excellent results without the need for any coupling coefficient. In unsteady FSI, the results coming from a model problem can also be used efficiently. However, each problem is different and new optimal coupling coefficients must then be estimated from the model problem.

\section{References}

[1] Perelman, T.L., "On conjugated problems of heat transfer," Int. J. Heat Mass Transfer 3, pp 293-303, 1961. https://doi.org/10.1016/0017-9310(61)90044-8.

[2] Luikov, A.V., Perelman, T.L., Levitin, R.S. and Gdalevich, L.B., "Heat transfer from a plate in a compressible gas flow, "Int. J. Heat Mass Transfer 13, 1971. https://doi.org/10.1016/0017-9310(70)90067-0.

[3] Roe, B., Jaiman, R., Haselbacher, A., Geubelle, P.H., "Combined interface boundary method for coupled thermal simulations, " International Journal for Numerical Methods in Fluids 57 (2008) 329-354.

[4] Henshaw, W.D., and Chand, K.K., "A composite grid solver for conjugate heat transfer in fluid-structure systems," Journal of Computational Physics 228 (2009) 3708-3741.

[5] Culler, D.J. and Mc Namara, J.J., "Studies on fluid-thermal-structural coupling for aerothermoelasticity in hypersonic flow," AIAA Journal 48 (8) (2010) 1721-1738.

[6] Lindström, J. and Nordström, J., "A stable and high-order conjugate heat transfer problem," Journal of Computational Physics 229 (2010) 5440-5456.

[7] He, L. and Oldfield, M.L.G., "Unsteady conjugate heat transfer modeling, " Journal of Turbomachinery 133 (3) (2011) 1022.1-10022.12.

[8] Sun, Z., Chew, J.W., Hills, N.J., Lewis, L. and Mabilat, C., "Coupled aerothermomechanical simulation for a turbine disk through a full transient cycle," Journal of Turbomachinery, no. 134, 2012. 
[9] Kazemi-Kamyab, V., van Zuijlen, A.H., Bijl, H., "Accuracy and stability analysis of a second-order timeaccurate loosely coupled partitioned algorithm for transient conjugate heat transfer problems," International Journal for Numerical Methods in Fluids 74 (2014) 113-133.

[10] Verstraete, T. and Scholl, S., "Stability analysis of partitioned methods for predicting conjugate heat transfer," International Journal of Heat and Mass Transfer 101 (2016), 852-869.

[11] Errera, M.P., and Chemin, S., "Optimal solutions of numerical interface conditions in fluid-structure thermal analysis," J. Comput. Phys. 245 (2013) 431-455. doi:10.1016/j.jcp.2013.03.004

[12] Errera, M.P., and Duchaine, F., Comparative study of coupling coefficients in Dirichlet-Robin procedure for fluid-structure aerothermal simulations," J. Comput. Phys. 312 (2016) 218-234. doi:10.1016/j.jcp.2016.02.022

[13] Godunov, S.K., and Ryabenkii, V.S., The theory of difference schemes: an introduction, North-Holland, Amsterdam, 1964 doi: $10.1126 /$ science.148.3671.811-a.

[14] El Khoury, R., Errera, M.P., El Khoury, K., Nemer, M., "Efficiency of coupling schemes for the treatment of steady state fluid-structure thermal interactions," Int. J. Therm. Sci. 115 (2017) 225-235. doi:10.1016/j.ijthermalsci.2017.02.001

[15] Felippa, C.A., and Park, K.C., "Staggered transient analysis procedures for coupled dynamic systems: formulation," Comput. Methods Appl. Mech. Eng. 24 (1980) 61-111. https://doi.org/10.1016/0045-7825(80)90040-7

[16] Le Tallec, P., "Domain decomposition methods in computational mechanics, " Comput. Mech. Adv. 1 (1994) 121-220.

[17] Piperno, S., Farhat, C., Larrouturou, B., "Partitioned procedures for the transient solution of coupled aroelastic problems Part I: Model problem, theory and two-dimensional application, " Comput. Methods Appl. Mech. Eng. 124 (1995) 79-112. https://doi.org/10.1016/0045-7825(95)92707-9

[18] Moretti, R., Errera, M.-P., Couaillier, V., and Feyel, F. "Stability, convergence and optimization of interface treatments in weak and strong thermal fluid-structure interaction," International Journal of Thermal Sciences, 126, pp 23-37, 2018.

[19] elsA : A CFD software package for compressible flows around complex various geometries. http://elsa.onera.fr

[20] Cambier, L., and Gazaix, M., "elsA : an efficient object-oriented solution to CFD complexity," AIAA Aerospace Science Meeting and Exhibit, AIAA 2002-0108, 2002. https://doi.org/10.2514/6.2002-108

[21] Cambier, L., Heib, S., and Plot, S., "The Onera elsA CFD software: input from research and feedback from industry, "Mech. \& Ind. 14 (2013) 159-174 doi:10.1051/meca/2013056

[22] Zset : Non-linear material \& structure analysis suite. http://www.zset-software.com

[23] CWIPI : Coupling with Interpolation Parallel Interface. http://sites.onera.fr/cwipi

[24] Errera, M.P., Lazareff, M., Garaud, J.D., Soubrié, T., Douta, C., and Federici, T., "A coupling approach to modeling heat transfer during a full transient flight cycle," Int. J. Heat Mass Transfer 110 (2017) 587-605. doi:10.1016/j.ijheatmasstransfer.2017.03.048 